\title{
Genome sequencing and analysis of admixed genomes of African and Mexican ancestry: implications for personal ancestry reconstruction and multi-ethnic medical genomics
}

Francisco M De La Vega ${ }^{1 *}$, Katarzyna Bryc ${ }^{2}$, Jeremiah D Degehnardt ${ }^{2}$, Shaila Musharoff ${ }^{2}$, Jeffrey M Kidd ${ }^{3}$, Vrunda Seth ${ }^{4}$, Sarah Stanley ${ }^{1}$, Abra Brisbin², Alon Keinan², Andrew Clark², Carlos D Bustamante ${ }^{2,3}$

From Beyond the Genome: The true gene count, human evolution and disease genomics

Boston, MA, USA. 11-13 October 2010

\section{Background}

Understanding the contribution of rare and common genetic variants to disease susceptibility will probably require multiand trans- ethnic sequencing studies that compare the genomes of many individuals with and without a particular disease. Accounting for the role of population stratification at fine scales, both in terms of genomic and geographic location, will be important because rare alleles are likely to show more population stratification. Here, we present results from sequencing, assembly and genomic analysis of two genomes from the Phase 3 HapMap. The donor individuals are of Mexican and African ancestry and represent the first 'admixed' genomes to be sequenced to high coverage.

\section{Materials and methods}

DNA was obtained from the Coriell Institute. We sequenced $2 \times 25 \mathrm{bp}$ and $2 \times 50 \mathrm{bp}$ mate-paired libraries with the Applied Biosystems SOLiD ${ }^{\mathrm{m}}$ System. The total average haploid coverage achieved was $\sim 20 \mathrm{X}$. Mapping and SNP calling was performed with the SOLiD analysis pipeline. We developed methods to estimate admixture breakpoints' along the admixed genomes.

\section{Results}

We used the distribution of admixture breakpoints to infer the personal admixture history of the samples and patterns of genomic diversity to reconstruct the demographic history of European, African and Native American continental populations. We compared the

'Genetic Systems R\&D, Life Technologies, Foster City, CA 94404, USA

Full list of author information is available at the end of the article distribution of functional and putatively neutral genetic variation among 12 sequenced genomes and found that difference in demographic history might account for statistically significant, differences in distributions of synonymous versus benign, possibly damaging, and probably damaging non-synonymous coding variants. Finally, we used the admixed genomes data together with 1000 Genomes to quantify the relative proportions of private, rare and common functional and neutral alleles within and among populations.

\section{Conclusions}

Genomic sequencing provides finer resolution of admixture breakpoints based on allele frequency estimates from HapMap and the 1000 Genomes Projects. Our results suggest that there are many new variants to be discovered in populations from African and the American descent, some of potential disease relevance.

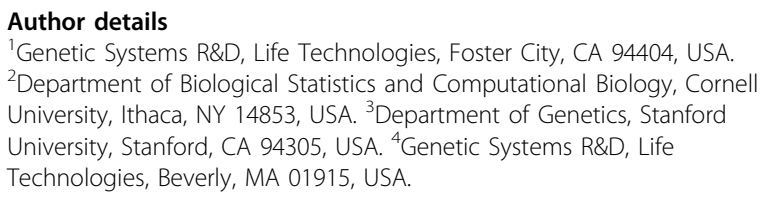

Published: 11 October 2010

doi:10.1186/gb-2010-11-S1-04

Cite this article as: De La Vega et al: Genome sequencing and analysis of admixed genomes of African and Mexican ancestry: implications for personal ancestry reconstruction and multi-ethnic medical genomics. Genome Biology 2010 11(Suppl 1):04. 